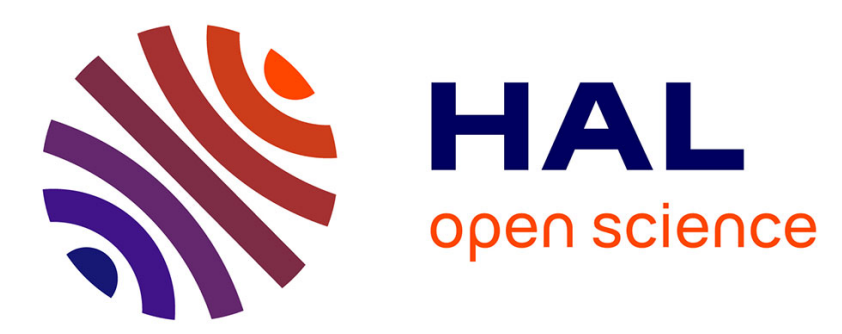

\title{
Metallic film optimization in a surface plasmon resonance biosensor by the extended Rouard method
}

Pierre Lecaruyer, Michael Canva, Jannick Rolland

\section{To cite this version:}

Pierre Lecaruyer, Michael Canva, Jannick Rolland. Metallic film optimization in a surface plasmon resonance biosensor by the extended Rouard method. Applied optics, 2007, 46 (12), pp.2361-2369. 10.1364/AO.46.002361 . hal-00664483

\section{HAL Id: hal-00664483 \\ https://hal-iogs.archives-ouvertes.fr/hal-00664483}

Submitted on 30 Jan 2012

HAL is a multi-disciplinary open access archive for the deposit and dissemination of scientific research documents, whether they are published or not. The documents may come from teaching and research institutions in France or abroad, or from public or private research centers.
L'archive ouverte pluridisciplinaire HAL, est destinée au dépôt et à la diffusion de documents scientifiques de niveau recherche, publiés ou non, émanant des établissements d'enseignement et de recherche français ou étrangers, des laboratoires publics ou privés. 


\title{
Metallic film optimization in a surface plasmon resonance biosensor by the extended Rouard method
}

\author{
P. Lecaruyer, M. Canva, and J. Rolland
}

\begin{abstract}
The extended Rouard method is applied to the computation of a multi-absorbing-layer system for the optimization of surface plasmon resonance (SPR) sensors. Specifically, the effect of the properties of a metallic layer on the shape of the reflectivity and sensitivity curve is demonstrated in the case of a Kretschmann configuration. This theoretical investigation allows us to establish the best optical properties of the metal to obtain a localized SPR, given the illuminating beam properties. Toward the development of a sensitive biosensor based on SPR, we quantify the changes in reflectivity of such an optical biosensor induced by the deposition of a nanometric biochemical film as a function of the metal film characteristics and the illumination operating conditions. The sensitivity of the system emphasizes the potential of such biophotonic technology using metallic multilayer configurations, especially with envisioned metamaterials. @ 2007 Optical Society of America
\end{abstract}

OCIS codes: $\quad 080.2720,310.6860,190.4350$.

\section{Introduction}

Surface plasmon resonance (SPR) has proved to be useful in the development of biosensors, specifically in the determination of biomolecular interactions. ${ }^{1-3}$ SPR is an optical sensing method used to measure small variations in refractive index in the vicinity of a thin metallic film. The phenomenon of SPR emerges from the existence of a plasmon wave. ${ }^{4-6}$ The plasmon wave is an induced effect of the coupling between a $p$ polarized (i.e., TM polarized) electromagnetic field and the oscillation of a plasma. Coupling is achieved under further particular illumination conditions characterized in part by the spectral characteristic of the source and the angle of incidence at which all the energy of the beam is transferred to the plasmon wave. Kretschmann proposed a prism-metal-air configuration to observe SPR, which we consider in this paper, except that the air layer is replaced by a dielectric

The authors are with the Laboratoire Charles Fabry de l'Institut d'Optique, CNRS, Université Paris-Sud, Campus Polytechnique, RD128, 91127 Palaiseau Cedex, France. E-mail address are, for P. Lecaruyer, pierre.lecaruyer@institutoptique.fr and, for M. Canva, michael.canva@iota.u-psud.fr. J. Rolland (jannick@odalab.ucf.edu) is on sabbatical leave from CREOL, College of Optics and Photonics, University of Central Florida, Orlando, Florida 32816, USA.

Received 27 July 2006; revised 7 December 2006; accepted 26 December 2006; posted 5 January 2007 (Doc. ID 73533); published 3 April 2007.

0003-6935/07/122361-09\$15.00/0

(C) 2007 Optical Society of America medium as illustrated in Fig. $1 .^{6}$ In such configuration the electromagnetic field and the plasma oscillation are represented by the optical wave of the illuminating beam and the oscillation of the charge density of the metallic layer, respectively. Given a TM-polarized collimated beam incident on the structure at a given angle of incidence and a wavelength that ensures phase matching between the parallel component of the wave vector of the incident beam and the wave vector of the surface plasmon wave, the energy can be transferred from the light beam to the plasmon mode. Hence the reflectivity of the incident beam through a Kretschmann configuration will drop to zero if the coupling is perfect between the two waves. As a consequence, a significant dip appears on the reflectivity curve, while we would have expected high reflectivity associated with total internal reflection.

Of interest for the application of biosensors are the evanescent waves propagating along the interface that are extremely sensitive to variations in the refractive index of the dielectric medium in the vicinity of the surface ${ }^{7}$ as well as to the metallic layer thickness and material properties. Variations in the dielectric medium may potentially be large, such as those induced by using different dielectric media, or small when induced by the bindings of molecules with the surface. Overall, variations of the stack structure have various effects on the reflectivity curve, such as dip translation, changes in the width and shape of the dip, and finally changes in the coupling efficiency, 


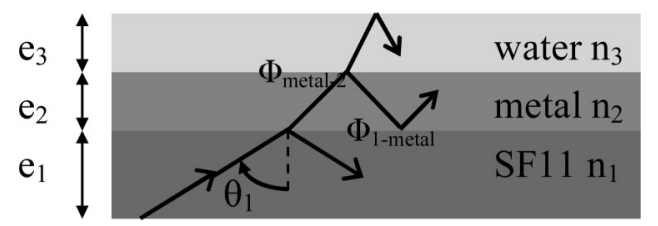

Fig. 1. Three layer stack representing the Kretschmann configuration. The metallic layer is located between the two dielectric media (i.e., SF11 and water) of refractive index $n_{1}$ and $n_{3}$ with $n_{1}>$ $n_{3}$. The phase difference arising with the reflection at an interface medium 1 /medium 2 is denoted $\Phi_{\text {medium 1-medium } 2 \text {. }}$

whose decrease would be demonstrated as a shallower dip in the reflectivity curve. The metallic layer properties are especially critical to the optimization of the stack structure. For a null or large thickness of the metallic layer, where large refers to thicknesses typically larger than $150 \mathrm{~nm}$, the multilayer configuration was previously shown to behave as a mirror. ${ }^{8}$ Therefore, between these extreme values, an optimum metallic thickness exists for maximum coupling efficiency.

We investigate in Section 2 the influence of the characteristics of a metallic layer on SPR. We first report the influence of the refractive index on the possibility of exciting the plasmon wave. We then specifically investigate the influence of the layer thickness on the coupling efficiency and on the optimization of the sensor sensitivity. Finally, we investigate the effects of the metallic index of refraction of theoretical materials and the effect of varying the wavelength on the shape of the SPR reflectivity curve, which allows us to quantify their impact on both the coupling efficiency and the sensitivity of the biosensor. In Section 3 we first justify the sensitivity metric for SPR-based biochip imaging that we utilize in our research. Finally, we investigate the influence of the stack structure parameters on the sensitivity of the biosensor, taking into account the necessity of multimetallic configurations to improve the chemical behavior and the capability of our experimental apparatus.

\section{Application of the Extended Rouard Method to the Characterization of Surface Plasmon Resonance}

To compare and analyze the different parameters involved in the SPR phenomenon and in particular the effect of the metallic layer characteristics in a multilayer stack, the reflectivity is computed by using the extended Rouard approach detailed in a recent publication. ${ }^{8}$ The advantage of this method lies in its simple implementation and generalization to as many layers (dielectric medium or metals) as necessary by the means of recursive loops.

A. Influence of the Metallic Layer Refractive Index on the Plasmon Wave Excitation

In this section we demonstrate the influence of the index of refraction of the metal on the shape of the plasmon curve through the consideration of different metals. To study the effect of the index of refraction of

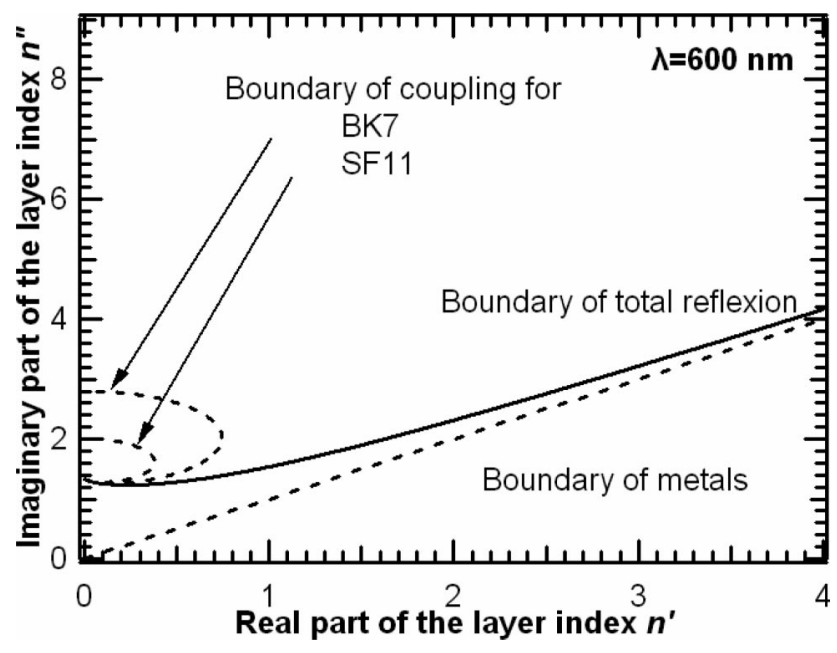

Fig. 2. Boundaries of the index of refraction $\left(n=n^{\prime}+i n^{\prime \prime}\right)$ of the layer inserted in order to observe the SPR in the case of a Kretschmann configuration glass-inserted layer-water. The longer dashed curve represents the asymptote of the next curve $\left(n^{\prime}=n^{\prime \prime}\right)$ for a refractive index with a very high real part; it represents the boundary for a material to be a metal. The solid curve (just above) represents the limit of the index of refraction of the inserted layer to yield total internal reflection. The short dashed curves (almost half circular) represent the limit of the refractive index of the inserted layer needed to excite the plasmon wave. The zone in which SPR occurs is delimited by the long solid curve and the appropriate short dashed curve.

the metal layer on SPR, the parameters of the illuminating beam were first fixed (i.e., angle of incidence and wavelength). A first study was completed by using theoretical indices of refraction for three different metals (i.e., gold, silver, and copper).

As the metal is an absorbing medium, indices of refraction of the metals in the form $n=n^{\prime}+i n^{\prime \prime}$ were considered. The importance of their real part $n^{\prime}$ and imaginary part $n^{\prime \prime}$ in achieving the SPR was investigated.

Results reported in Fig. 2 show the boundaries of the zone where SPR occurs in terms of indices of refraction of the metallic layer. The zone where the SPR occurs is delimited by two curves that represent the required, conditions for exciting the plasmon wave. First, the condition of excitation requires a total internal reflection without any metallic layer, which means considering Snell's law that

$$
n_{\text {prism }} \sin \theta_{\text {prism/dielectric }}>n_{\text {dielectric }} .
$$

Moreover, the condition of excitation of the plasmon requires that the projection of the wave vector of excitation equal the component of the plasmon wave vector, which means in terms of refractive index that

$$
n_{\text {prism }}>n_{\text {effective index }}
$$

where $n_{\text {effective index }}$ represents the index of the guide of the plasmon wave. $n_{\text {effective index }}$ is obtained from the wave vector $k_{\text {SPR }}$ given by ${ }^{7,10}$ 


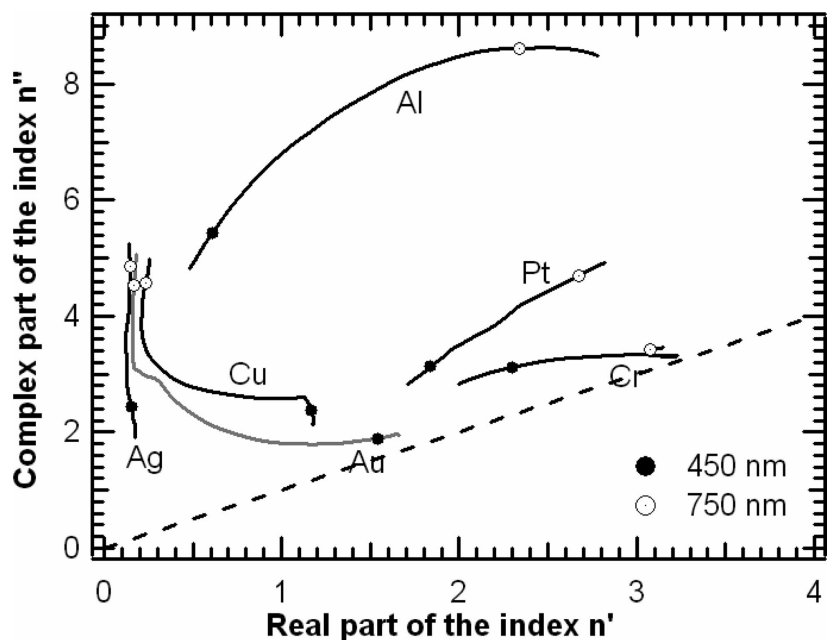

Fig. 3. Trajectories of six metals in the complex plan of index of refraction from 400 to $800 \mathrm{~nm}$. The dashed line represents the boundary for which $n^{\prime}<n^{\prime \prime}$.

$$
k_{\mathrm{SPR}}=\frac{2 \pi}{\lambda} \sqrt{\frac{n_{m}{ }^{2} n_{d}{ }^{2}}{{n_{m}}^{2}+{n_{d}}^{2}}},
$$

where $n_{m}$ and $n_{d}$ represent the indices of refraction of the metal and of the cover dielectric medium, respectively, and $\lambda$ is the wavelength of the illuminating beam. This condition is represented by the boundary $\left(n_{\text {prism }}=n_{\text {effective index }}\right)$, the solid curve in Fig. 2. Considering the effective index of the plasmon, according to Eq. (1)

$$
n_{\text {effective index }}>n_{\text {dielectric }} \text {. }
$$

This condition is represented by the almost halfcircular boundaries and depends on the type of glass prism used in the calculation.

Results reported in Fig. 3 show the trajectories of the six metals (silver, gold, copper, chromium, platinum, aluminum) represented in the complex plane in the visible range from 400 to $800 \mathrm{~nm}$. Two sets of dots (i.e., filled and open circles, corresponding to 450 and $750 \mathrm{~nm}$, respectively) were added to show the evolution of the curve as a function of the wavelength, and the associated coordinates are reported in Table 1. These curves were obtained by smoothing spline interpolation of SOPRA's data for the metals and from Schiebener's data for water. ${ }^{11,12}$

\section{B. Optimization of the Metallic Thickness Based on Coupling Efficiency}

Even though it is possible to excite the plasmon wave, there is only one thickness associated with a configuration and a wavelength that allows an optimized transfer of energy from the illuminating beam to the plasmon wave. ${ }^{13,14}$

To have a propagating wave in the metallic layer, we must have constructive interference, which means a phase difference equal to 0 for each path after two
Table 1. Index of Refraction of Six Metals for Two Wavelengths

\begin{tabular}{cccc}
\hline Metal & $\lambda(\mathrm{nm})$ & $n^{\prime}$ & $n^{\prime \prime}$ \\
\hline $\mathrm{Ag}$ & 450 & 0.1546 & 2.4364 \\
& 750 & 0.1468 & 4.8604 \\
$\mathrm{Au}$ & 450 & 1.5397 & 1.8927 \\
& 750 & 0.1676 & 4.5332 \\
$\mathrm{Cu}$ & 450 & 1.1681 & 2.3783 \\
& 750 & 0.2321 & 4.5639 \\
$\mathrm{Pt}$ & 450 & 1.8378 & 3.1273 \\
& 750 & 2.6718 & 4.6965 \\
$\mathrm{Al}$ & 450 & 0.6083 & 5.4265 \\
& 750 & 2.3355 & 8.6158 \\
$\mathrm{Cr}$ & 450 & 2.2984 & 3.1193 \\
& 750 & 3.0734 & 3.4146 \\
\hline
\end{tabular}

reflections on the interfaces illustrated in Fig. $1 .^{15}$ Therefore, without any assumption about the complex nature of the different terms of the expression, we have in the case of one metallic layer, inferred from Fig. 1,

$$
\frac{4 \pi n_{\text {metal }} e_{\text {metal }} \cos \theta}{\lambda}+2 \Phi_{1-\text { metal }}+2 \Phi_{\text {metal }-3}=2 m \pi,
$$

where $\Phi_{i \text { metal }}=\arctan \left[\left(n_{\text {metal }} \sqrt{n_{\text {metal }}^{2} \sin ^{2} \theta-n_{i}^{2}}\right) /\left(n_{i}^{2}\right.\right.$ $\cos \theta)]$ and $m$ is an integer.

From Eq. (5), the optimum metallic thickness for an optimum coupling coefficient is given as

$$
e_{\text {metal }}=\left|\frac{\lambda\left(\Phi_{1-\text { metal }}+\Phi_{2-\text { metal }}\right)}{2 \pi n_{\text {metal }} \cos \theta}\right| .
$$

There is still no assumption about the complex nature of the metallic layer thickness, which is a function of $\theta$. However, since a thickness should be real, the optimum thickness is obtained from the real part of $e_{\text {metal }}$ for an angle $\theta$ that is associated with a null imaginary part.

In Fig. 4, the curve represents the minimum reflectivity as a function of the metallic layer thickness at $750 \mathrm{~nm}$ and for an optimized associated angle of incidence in an SF11- silver layer-water configuration. Note that in the range of good coupling, limited by the full width at half-maximum (FWHM) of the dip, the optimized associated angle of incidence remains almost constant around $51.3^{\circ} \pm 0.3^{\circ}$ with or without the additional $1 \mathrm{~nm}$ of dielectric as shown in Fig. 5a below and further motivated and detailed in subsection 2.C. A minimum appears in Fig. 4, which represents the optimum thickness for a maximized coupling coefficient. From Fig. 4 the optimum thickness of a silver film is $47 \mathrm{~nm}$ at a wavelength of $750 \mathrm{~nm}$. Note that a perfect coupling might not exist, depending on the metallic layer and the illuminating beam properties. 


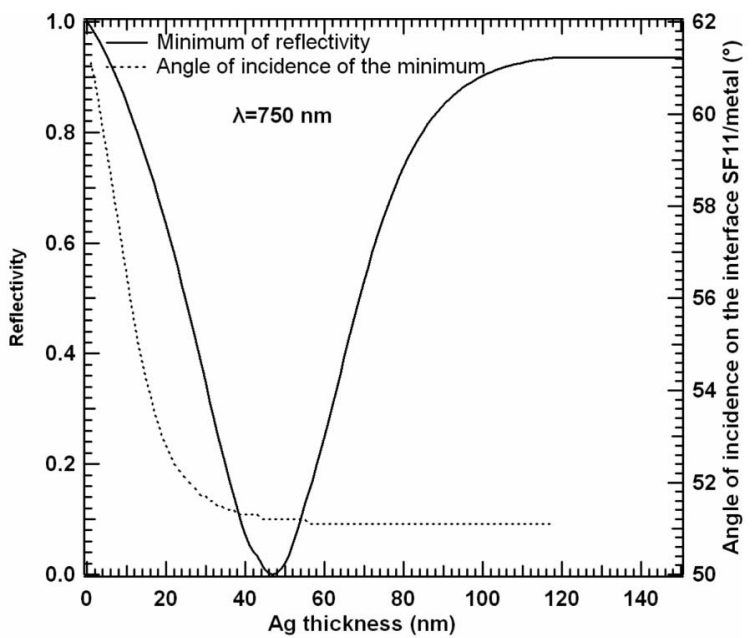

a

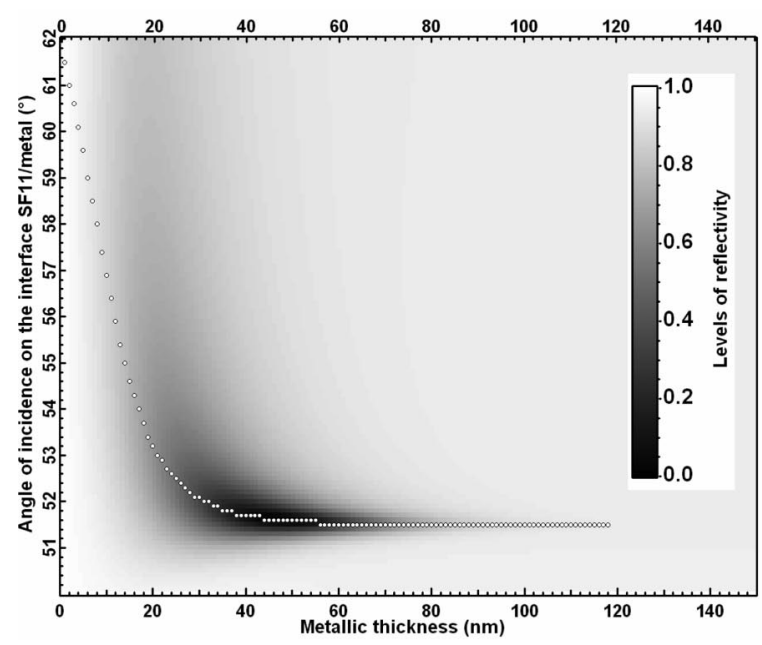

b

Fig. 4. a, Reflectivity curve of the minimum reflectivity as a function of the silver thickness in an SF11-silver-water configuration at $750 \mathrm{~nm}$; with the optimized associated angle of incidence, a minimum appears for a thickness of $47 \mathrm{~nm}$, representing the best coupling coefficient. b, Surface plot of the reflectivity as a function of the angle of incidence and the metallic thickness. The trajectory shows clearly that the resonance angle changes as the metallic thickness changes.

\section{Influence of the Thickness of the Metallic Layer on Sensitivity}

As already explained in Subsection 2.B, at a fixed wavelength and for each metal an optimum thickness exists that maximizes the coupling coefficient. Therefore it is interesting to investigate whether this optimum thickness can be associated with an optimum sensitivity. The configuration we consider in this section remains an SF11-silver-water system (cover medium) at the wavelength of $750 \mathrm{~nm}$.

The angular reflectivity curve was first calculated for the configuration SF11-silver-water at a fixed metallic thickness. We thus calculated the angular reflectivity curve after the insertion of a DNA layer simulated by $1 \mathrm{~nm}$ of dielectric of index $n=1.46$ (as measured for dense layers ${ }^{16}$ ) between the silver layer

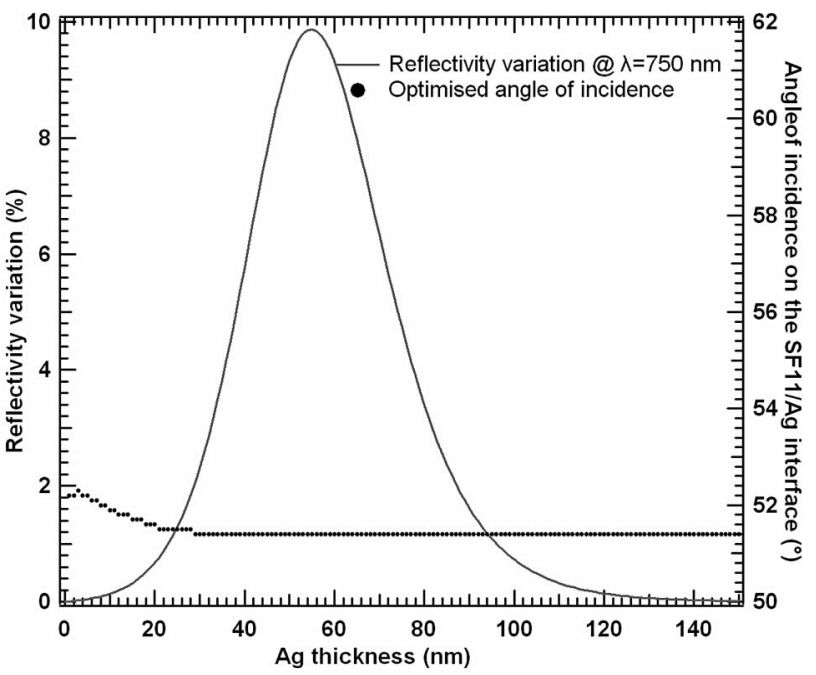

a

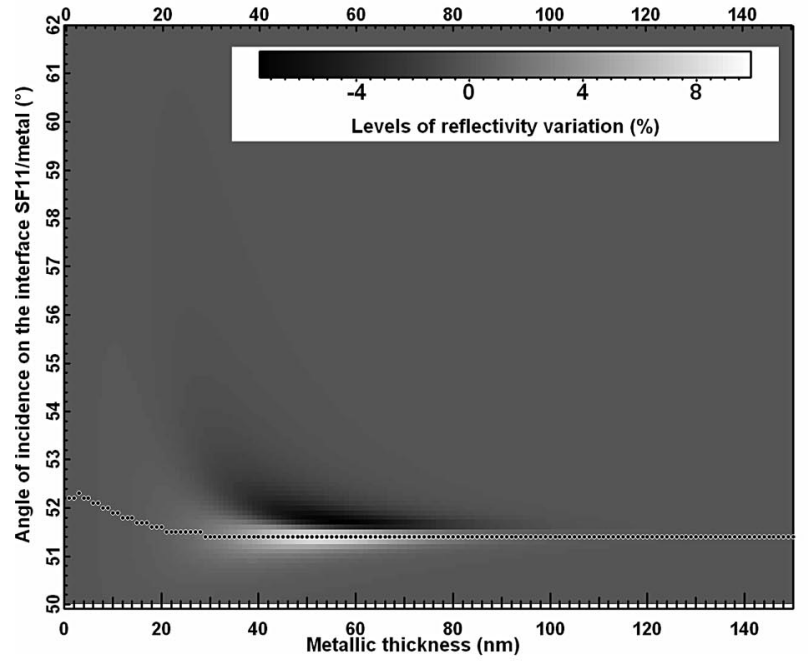

b

Fig. 5. a, Maximum reflectivity variation curve between an SF11silver-water configuration and an SF11-silver-DNA layer-water configuration as a function of the silver thickness at $750 \mathrm{~nm}$; a maximum appears for a thickness of $54 \mathrm{~nm}$, representing the best transducing coefficient. The parameters of the homogeneous DNA layer are $n=1.46$ and thickness $1 \mathrm{~nm}$. b, Surface plot of the reflectivity variation as a function of the angle of incidence and the metallic thickness, illustrating that the optimum thickness for maximized coupling and maximized sensitivity are slightly different.

and the cover medium. Figure 5 shows the maximum of the angular differential curve between the two former configurations, with DNA layer and without DNA layer, as a function of the metallic thickness $e_{\text {metal }}$. We calculated that the sensitivity has a maximum for $e_{\text {metal }}=54 \mathrm{~nm}$, which is slightly higher than the thickness corresponding to the maximized coupling coefficient as first shown in Fig. 4.

D. Influence of the Metallic Layer Refractive Index on the Shapes of the Reflectivity and Reflectivity Variation Curves The theoretical growth of an additional layer of constant index of refraction equal to 1.46 and thickness 
$1 \mathrm{~nm}$ was next computed to simulate the effect of the deposition of biomolecules on a metallic surface. The effect of variations of the index of refraction of the metallic layer on the shape of the reflectivity curve is demonstrated in order to simulate the effect of the insertion of molecules into a dielectric medium with different metallic configurations. In this subsection the term "sensitivity" is used to represent the reflectivity variation after the insertion of $1 \mathrm{~nm}$ of the intermediary layer between the metal layer and the water medium.

To investigate the influence of the refractive index of the metallic layer on the shape of the plasmon reflectivity curve, the plasmon curves of four Kretschmann configurations with imaginary materials were studied. Those four materials responded to four couples of refractive index $n=n^{\prime}+i n^{\prime \prime}$ with $n^{\prime}=0.1,0.2$ and $n^{\prime \prime}=2.0,4.0$. The optimum thicknesses were calculated by using the same method as in the previous subsection to superimpose and compare the reflectivity curves to one another. Figure $6 \mathrm{a}$ illustrates that as the complex part $n^{\prime \prime}$ becomes larger (i.e., from 2.0 to 4.0 ), the dip becomes significantly narrower. When $n^{\prime \prime}$ varies from to 2 to 4 , the FWHM of the dip varies from around $16.7^{\circ}$ to $1^{\circ}$ and from $21.6^{\circ}$ to $2.3^{\circ}$ for $n^{\prime}=0.1$ and $n^{\prime}=0.2$, respectively. Similarly, as the real part of the refractive index $n^{\prime}$ becomes smaller, the dip becomes narrower. When $n^{\prime}$ varies from to 0.2 to 0.1 , the FWHM of the dip varies from around $2.3^{\circ}$ to $1^{\circ}$ and from $21.6^{\circ}$ to $16.7^{\circ}$ for $n^{\prime \prime}=4$ and $n^{\prime \prime}=2$, respectively. In Fig. $6 \mathrm{~b}$ the sensitivity curves of the four Kretschmann configurations (SF11-imaginary materials-water) were computed for the absorption (insertion) of $1 \mathrm{~nm}$ of a dielectric with refractive index $n=1.46$. In this figure it is also clear that as $n^{\prime \prime}$ becomes larger, the sensitivity curve is more localized relative to the angle of incidence, and the maximum becomes higher. In the same way, as $n^{\prime}$ becomes smaller, the maximum of the angular reflectivity variation curve becomes higher. From those four couples, at a fixed wavelength $(600 \mathrm{~nm})$, it can be seen that the maximum of the sensitivity curve can be associated with the narrowness of the dip of the plasmon curve. This finding signifies that the sensitivity (proportional to the reflectivity variation curve) is increased as $n^{\prime}$ becomes smaller and $n^{\prime \prime}$ becomes higher.

From the trajectories of the metals in the $\left(n^{\prime}, n^{\prime \prime}\right)$ complex plan and the assumptions made above, three Kretschmann configurations with three metals (sil-

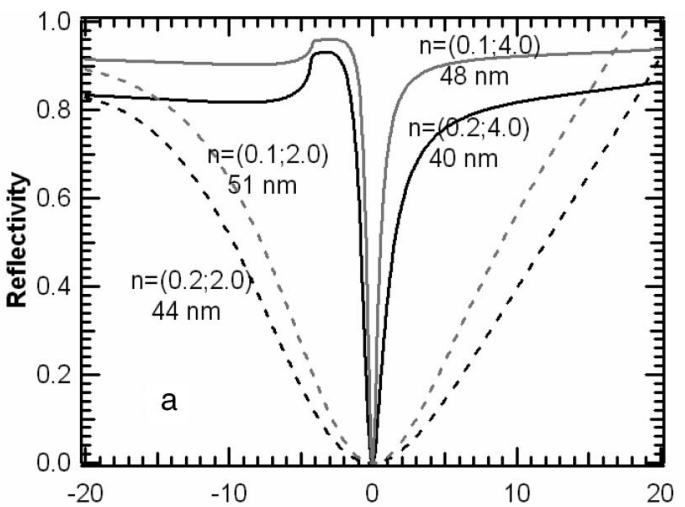

Differential angle of incidence on SF11/metal interface

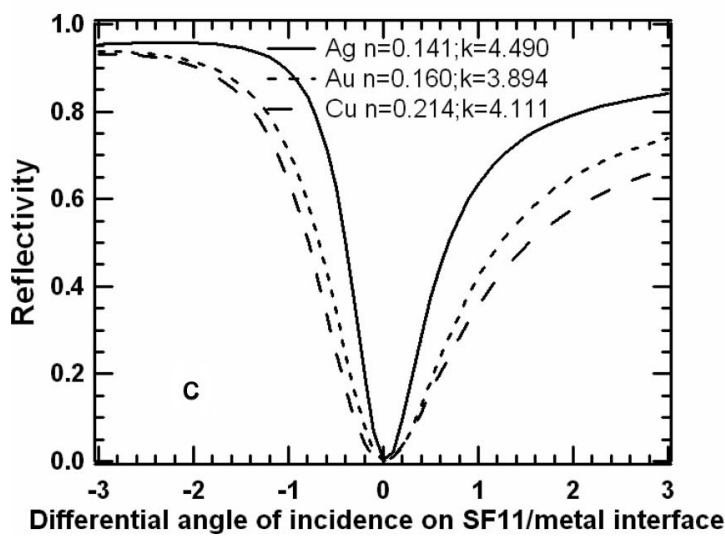

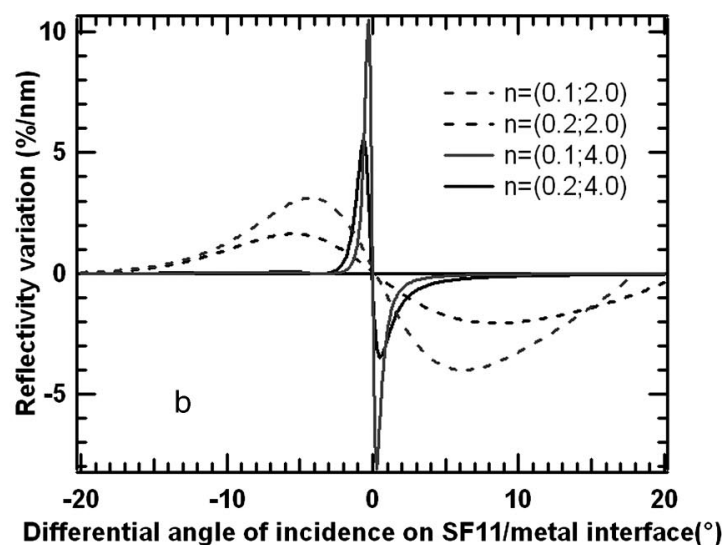

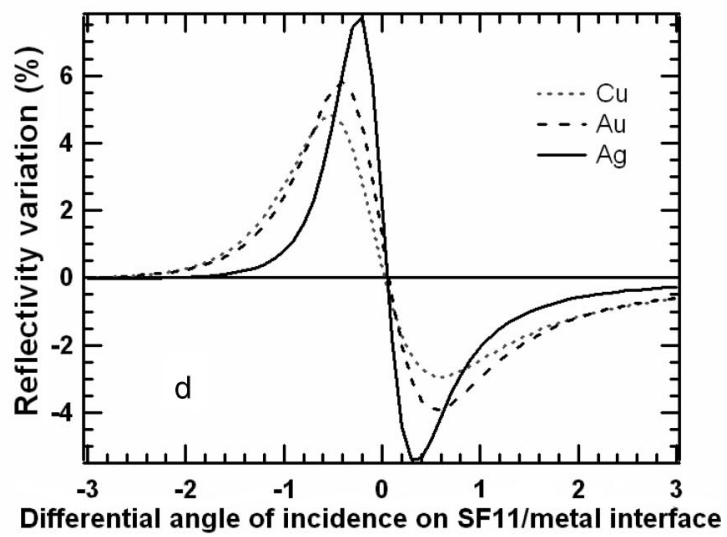

Fig. 6. a, Angular reflectivity curves at $600 \mathrm{~nm}$ centered around $0^{\circ}$ for four materials with optimized coupling thicknesses and offsets in an SF11-material-water configuration, demonstrating the influence of $n^{\prime}$ and $n^{\prime \prime}$ on the narrowness of the absorption dip. b, Angular reflectivity variation curves at $600 \mathrm{~nm}$ centered around 0 after the insertion of a $1 \mathrm{~nm}$ layer of index of refraction $(n=1.46)$. c, Angular reflectivity curves at $700 \mathrm{~nm}$ centered around $0^{\circ}$ for three metals with different thicknesses (silver, $e=54 \mathrm{~nm}$; gold, $e=51 \mathrm{~nm}$; copper, $e=45 \mathrm{~nm}$ ) and angular offsets in an SF11-metal-water configuration. d, Angular reflectivity variation curves at $700 \mathrm{~nm}$ centered around 0 after the insertion of a $1 \mathrm{~nm}$ layer of index of refraction $(n=1.46)$. 
ver, gold, and copper) were chosen. The parameters of those materials are for $\mathrm{Ag}, n^{\prime}=0.141, n^{\prime \prime}=4.490$, $e=47 \mathrm{~nm}$; gold, $n^{\prime}=0.160, n^{\prime \prime}=3.894, e=51 \mathrm{~nm}$; copper, $n^{\prime}=0.214, n^{\prime \prime}=4.111, e=45 \mathrm{~nm}$. In Fig. $6 \mathrm{c}$ the plasmon reflectivity curves of the SF11-prismmetal-water configurations are represented as a function of the angle of incidence (with an offset to center the three curves around 0) at $700 \mathrm{~nm}$. From those curves, the influence of $n^{\prime}$ and $n^{\prime \prime}$ on the shape of the reflectivity curves is confirmed. The narrowest dip of SPR is obtained from the silver configuration, which has the smallest $n^{\prime}$ and the highest $n^{\prime \prime}$ of the three metals. In Fig. 6d, as expected, the highest sensitivity curve for the insertion of $1 \mathrm{~nm}$ of dielectric with index $n=1.46$ is obtained from the silver configuration. Moreover, it is clear that for a fixed wavelength, as the dip becomes narrow, the sensitivity to the insertion of $1 \mathrm{~nm}$ of dielectric is increased.

Given that the best metal for SPR requires a small real part $n^{\prime}$ and a large imaginary part $n^{\prime \prime}$ of the refractive index to get a localized dip in terms of the illuminating beam parameters, the silver is preferred, as shown in Fig. 3.


Fig. 7. a, Angular reflectivity curves of an SF11-silver layerwater configuration for two sets of parameters $(\lambda=450 \mathrm{~nm}, e=$ $40 \mathrm{~nm} ; \lambda=750 \mathrm{~nm}, e=47 \mathrm{~nm}$ ) with the insertion of $1 \mathrm{~nm}$ of dielectric $(n=1.46)$ and without this dielectric. b, Optimized angular reflectivity variation curves after the insertion of $1 \mathrm{~nm}$ of dielectric $(n=1.46)$ in an SF11-silver layer-water configuration $(\lambda=450 \mathrm{~nm}, e=45 \mathrm{~nm} ; \lambda=750 \mathrm{~nm}, e=54 \mathrm{~nm})$.
E. Wavelength Influence on the Shape of Reflectivity and Reflectivity Variation Curves

From the trajectories of the metals and from the evolution of the refractive index of silver, the real part $n^{\prime}$ of the refractive index for silver is almost constant, as shown in Table 1 , while the imaginary part $n^{\prime \prime}$ becomes twice as large as the wavelength increases from 450 to $750 \mathrm{~nm}$. For each wavelength, the optimum thicknesses are calculated in order to have the best coupling coefficient.

In Fig. $7 \mathrm{a}$, as the wavelength increases from 450 to $750 \mathrm{~nm}$, the dip becomes narrower and the angular shift for the insertion of $1 \mathrm{~nm}$ of dielectric is higher for $450 \mathrm{~nm}$ than for $750 \mathrm{~nm}$. The variation of the complex part $n^{\prime \prime}$ with wavelength explains the narrowing of the dip of absorption as the imaginary part becomes larger.

In Fig. 7b, as expected, since $n^{\prime}$ remains almost constant from 450 to $750 \mathrm{~nm}$ and $n^{\prime \prime}$ becomes twice as large, the maximum reflectivity variation associated with the insertion of $1 \mathrm{~nm}$ of dielectric is obtained from the $750 \mathrm{~nm}$ sensitivity curve with a maximum reaching nearly $9.2 \%$.

Considering the evolution of the various trajectories of the metals in the visible range, we have quantified how the sensitivity increases with the wavelength for the three metals chosen in the Subsection 2.D (silver, gold, and copper).

In this section the parameters of the metallic layer have been investigated and their importance has been demonstrated. From these results the optimization of the metallic thickness to yield the most sensitive Kretschmann configuration is possible.

\section{Sensitivity of the Biosensor}

Some SPR sensors are built to detect the position of the minimum of the reflectivity curve. In this case the importance of having an extremely narrow dip is naturally critical for the accuracy of the measurement of this minimum. Two different definitions of sensitivity emerged from this way of measurement and are detailed in recent publications. ${ }^{17-19}$ The first one is based on the angle of incidence shift induced by a variation of the index of the analyte. The second one is based on the wavelength shift induced by a variation of the index of the analyte.

In the case of the SPR imaging sensor developed in our laboratory, the aim is to have an optical sensor sensitive to the insertion of a subnanometric biological layer in the multilayer system between the metal and the dielectric cover medium or, for other applications, to variations in the index of refraction of the aqueous solution (also called buffer) up to an order of about $10^{-5}$. Therefore the definition of the sensitivity used in our work is based on the variation of reflectivity induced by the insertion of a layer of $1 \mathrm{~nm}$ (index of refraction $n=1.46$ ) in our Kretschmann configuration between the metallic layer and the semi-infinite dielectric medium.

In quantifying the sensitivity of the technique, in this case we are measuring the slope of the plasmon 
curve on the ascent from the dip because the change in slope is a lot more sensitive than the change in the dip location and also more robust because of the linearity of the response over a large range of the source characteristics (i.e., wavelength and angle of incidence).

We have seen that the slope becomes steeper as the dip becomes narrower. However, it is also important to have not only a narrow dip, since the sensitivity is directly linked to the slope of the plasmon curve, but also a large dip translation, since the sensitivity is the optimized combination between the translation and the slope.

In this section no investigation is performed on the sensitivity to biological layer parameters (i.e., thickness and refractive index). Indeed, with our setup, to first order the reflectivity variation $(\Delta R)$ remains almost constant with a constant product of the thickness $e$ of the inserted dielectric layer and the refractive index variation $\Delta n\left(\Delta n=n_{\text {dielectric }}-n_{\text {water }}\right)$. Therefore, for a small change of one of the biological layer parameters, we can consider that a rule of three can be applied to calculate the associated sensitivity.

Although studies performed in Section 2 showed that the silver would be the best metal choice in the visible range from 450 to $750 \mathrm{~nm}$, with gold as a close follower: the gold is usually preferred because of its best chemical stability (i.e., no oxidation) in contact with aqueous solution and also because of the wellknown and widely studied gold-thiol chemical interaction. Since silver and gold are the best metals for maximum sensitivity, the sensitivity of an SF11silver/gold couple-water configuration can be computed to benefit from the high sensitivity of silver and chemical stability of gold.

In Fig. 8a the sensitivity for the insertion of $1 \mathrm{~nm}$ of dielectric is depicted as a function of the thicknesses of silver and gold at $600 \mathrm{~nm}$. As expected, since the silver configuration is more sensitive, for a fixed thickness of the couple silver/gold, the sensitivity increases as the ratio thickness of silver versus thickness of gold increases. The maximum sensitivity is thus reached for a null thickness of gold and is up to $9 \%$. However, to use this configuration combining the advantages of the two metals, assuming a minimum thickness of gold of $2 \mathrm{~nm}$, a sensitivity up to $8.35 \%$ is achievable, compared with only $3.49 \%$ for just gold.

Unfortunately, the silver, like gold, has a poor adherence with glass; therefore chromium or titanium must be inserted to fix the silver or gold layer to the glass prism. ${ }^{20}$ From the trajectories of the indexes of refraction of chromium, we expect a loss in sensitivity. In Fig. 8b, the sensitivity for the couple chromium/ gold drops to a maximum of $3.49 \%$ and to around $3.25 \%$ for a minimum thickness of chromium of $2 \mathrm{~nm}$, which is the minimum thickness that seems possible experimentally for good binding of gold.

In Fig. 8c, to benefit from the adherence of chromium, from the sensitivity of silver, and from the chemical advantages of gold, a configuration with

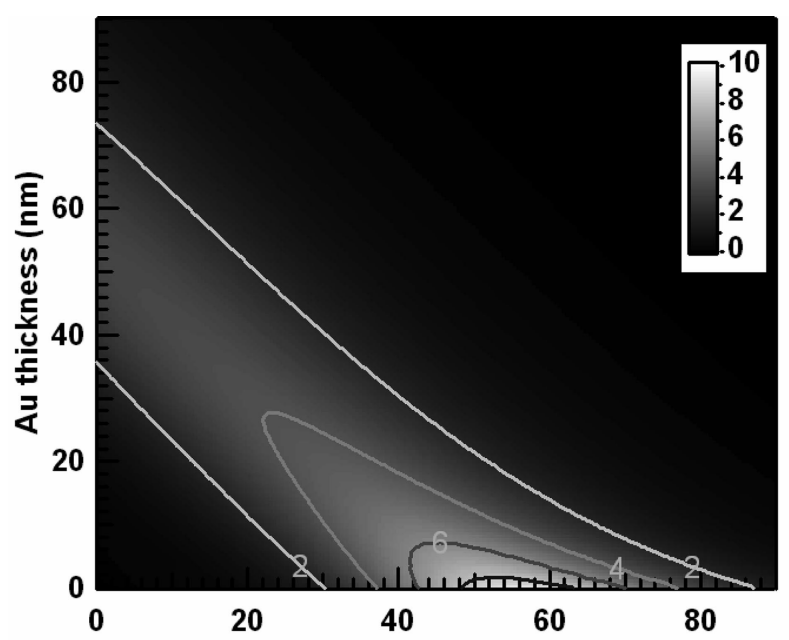

a

Ag thickness $(\mathrm{nm})$
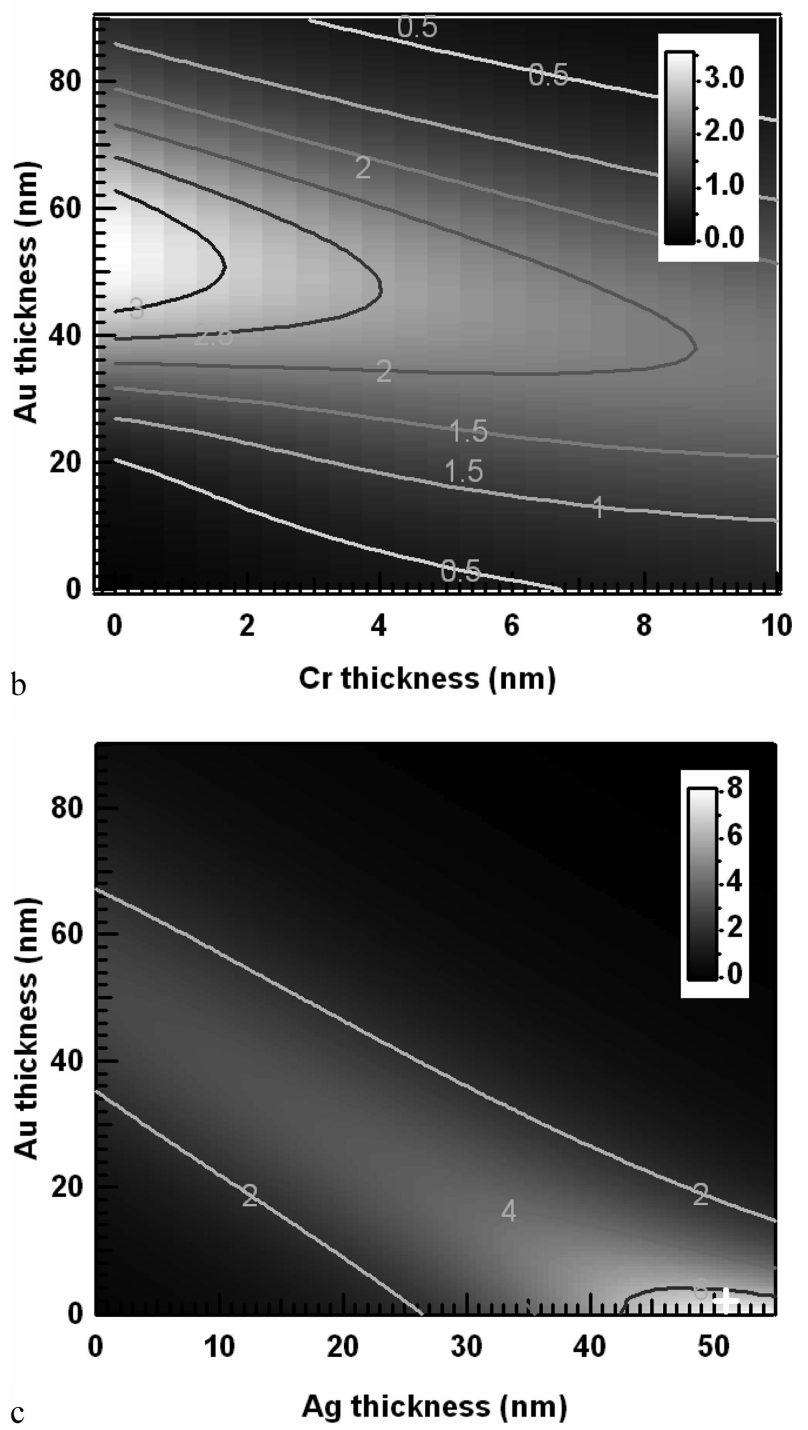

Fig. 8. Maximum reflectivity variation for the insertion of $1 \mathrm{~nm}$ of dielectric $(n=1.46)$ as a function of the thicknesses of a combination of metals ( $a$, silver-gold; b, chromium-gold; c, chromiumsilver-gold) at $600 \mathrm{~nm}$. The white cross represents the maximum reflectivity variation for an SF11-chromium-silver-gold-water configuration with $2 \mathrm{~nm}$ of chromium and gold. 
$2 \mathrm{~nm}$ of chromium combined with various thicknesses of silver and gold has been investigated for sensitivity. Inserting the silver layer into the former configuration at $600 \mathrm{~nm}$ can increase the sensitivity from $3.49 \%$ to more than $6.7 \%$ with $2 \mathrm{~nm}$ of gold to cover the silver layer (represented by a white cross in Fig. 8c). This means that the sensitivity could be almost doubled at a fixed wavelength.

As new optical properties are possible nowadays through the discovery of new structures such as metamaterials, ${ }^{21}$ it is interesting to compute the optimum sensitivity of a material at $600 \mathrm{~nm}$ knowing $n^{\prime}$ and $n^{\prime \prime}$ for the detection of $1 \mathrm{~nm}$ of dielectric of index $n=1.46$. Figure 9a shows the maximum sensitivity as a function of the index $n^{\prime}$ and $n^{\prime \prime}$ of the hypothetical metamaterial inserted into the Kretschmann configuration. By adjusting the proper thickness for the best coupling coefficient, it is observed that it might be possible to reach a sensitivity of more than $85 \%$ with $n^{\prime}$ as small as possible (0.01) and $n^{\prime \prime}$ large (more

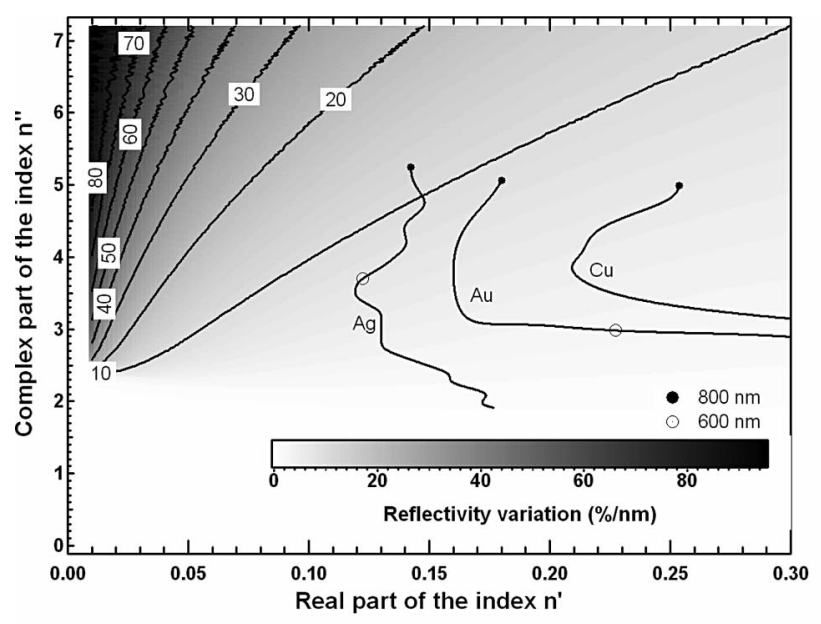

a

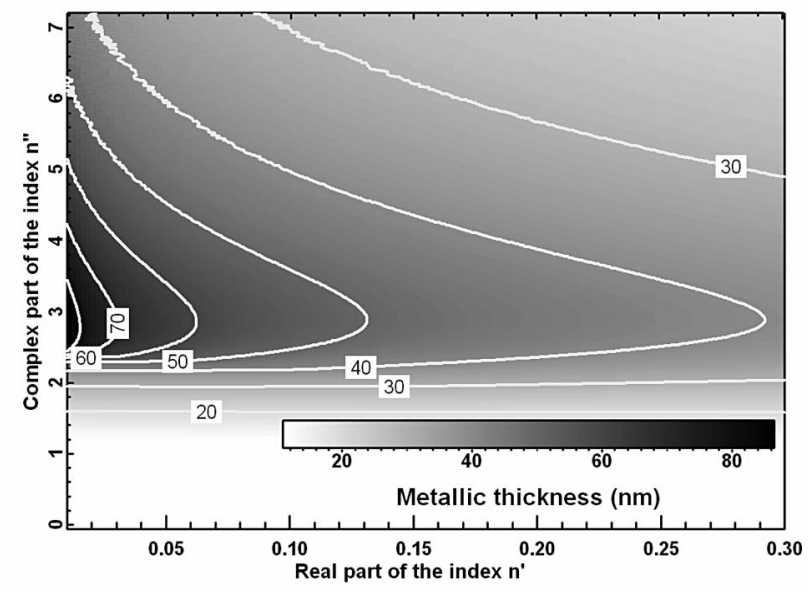

b

Fig. 9. a, Surface plot of the maximum reflectivity variation obtained for an SF11-layer of index $n=n^{\prime}+i n^{\prime \prime}$-water configuration after the deposition of $1 \mathrm{~nm}$ of dielectric of index $n=1.46$. b, Surface plot of the associated optimum thickness of the inserted metallic layer. than 6.7). This study shows that the evolution of SPR sensors can still be greatly improved.

However, the system used in our laboratory is limited by the metallic thickness attainable and the mechanical parameters of the apparatus such as the angular accuracy attainable. Thus, if the absorption dip is too narrow as a function of the metallic thickness, such as defined in Fig. 4, a stable coupling coefficient might not be possible throughout the surface of the sensor. The sensitivity in some areas of the surface would be dramatically lowered. Moreover, if the absorption dip is too localized in terms of the angle of incidence, the FWHM of the absorption dip may be too small to ensure a near-optimal response in terms of the sensitivity of the apparatus throughout the surface of the sensor.

From previous experiments performed in the laboratory, our apparatus (system+biochip) has an angular dispersion of the SPR of around $0.3^{\circ} .{ }^{22,23}$ Therefore, if the angular FWHM of the dip is lower than the angular dispersion of the apparatus, the sensitivity of $85 \%$ would have no applicability.

From those two essential parameters for the stability of the apparatus, a metamaterial with an index of refraction of around $n \sim 0.01+i 3.5$ would be a good compromise that would give a theoretical reflectivity variation of about $40 \% \mathrm{~nm}^{-1}$ compared with a few percent currently achieved.

\section{Conclusions}

The Rouard method extended to absorbing media was successfully applied to the modeling of surface plasmon resonance and particularly to the computation of reflectivity through a Kretschmann configuration. In investigating the effect of the optical properties of a metallic layer on the coupling of an illuminating beam with a plasmon wave, it was shown that the best metal to achieve efficient coupling and a localized SPR as a function of the angle of incidence had to be as close as possible to a metal with a solely imaginary index of refraction and that the optimal thickness of the metal was highly dependent on the characteristics of the illuminating beam.

In the application to biosensors, the detection of interactions can be made by measuring variations of optical signals that, in our case, correspond to variations in the reflectivity of a multiple-layer stack. Computations of the sensitivity of the sensor as a function of the characteristics of a realistic metallic multilayer Kretschmann configuration (silver/gold) showed sensitivity improvements of about a factor of 2. Computing the sensitivity as a function of the index of refraction of a metamaterial also showed that these sensors still have a great potential to improve in sensitivity with the discovery of new structures.

\section{References}

1. B. Liedberg, C. Nylander, and I. Lundström, "Surface plasmon resonance for gas detection and biosensing," Sens. Actuators 4, 299-304 (1983).

2. B. Rothenhausler and W. Knoll, "Surface plasmon microscopy," Nature 332, 615-617 (1988). 
3. J. M. McDonnell, "Surface plasmon resonance: towards an understanding of the mechanisms of biological molecular recognition," Curr. Opin. Chem. Biol. 5, 572-577 (2001).

4. U. Fano, "The theory of anomalous diffraction gratings and of quasi-stationary waves on metallic surfaces (Sommerfeld's waves)," J. Opt. Soc. Am. 31, 213-222 (1941).

5. A. Otto, "Excitation of surface plasma waves in silver by the method of frustrated total reflection," Z. Phys. (Leipzig) 216, 398-410 (1968).

6. E. Kretschmann and H. Raether, "Radiative decay of nonradiative surface plasmons excited by light," Z. Naturforsch. 23A, 2135-2136 (1968).

7. H. Raether, Surface Plasmons on Smooth and Rough Surfaces and on Gratings (Springer-Verlag, 1988), pp. 4-18, 125-126.

8. P. Lecaruyer, E. Maillart, M. Canva, and J. Rolland, "Generalization of the Rouard method to an absorbing thin film stack and application to surface plasmon resonance," Appl. Opt. 45, 8419-8423 (2006).

9. M. P. Rouard, "Etudes des propriétés optiques des lames métalliques très minces," Ann. Phys. (Paris) Ser. II 7, 291-384 (1937).

10. J. Ctyroky, J. Homola, P. V. Lambeck, S. Musa, H. J. W. M. Hoekstra, R. D. Harris, J. S. Wilkinson, B. Usievich, and N. M. Lyndin, "Theory and modelling of optical waveguide sensors utilising surface plasmon resonance," Sens. Actuators B 54, 66-73 (1999).

11. Available at http://www.sopra-sa.com/index2.php?goto= $\mathrm{d} 1 \& \mathrm{rub}=4$.

12. P. Schiebener, J. Straub, J. M. H. Levelt-Sengers, and J. S. Gallagher, "Refractive index of water and steam as function of wavelegth, temperature and density," J. Phys. Chem. Ref. Data 19, 677-717 (1990).

13. B. H. Ong, X. Yuan, S. C. Tjuin, J. Zhang, and H. M. Ng, "Optimized film thickness for maximum field enhancement of a bimetallic surface plasmon resonance biosensor," Sens. Actuators B 114, 1028-1034 (2006).

14. S. Y. Wu and H. P. Ho, "Sensitivity improvement of the surface plasmon resonance optical sensor by using a gold-silver transducing layer," 2002 IEEE Hong Kong Electron Devices Meeting (IEEE, 2002), pp. 63-68.

15. M. Born and E. Wolf, Principles of Optics: Electromagnetic Theory of Propagation, Interference and Diffraction of Light (Pergamon, 1959).

16. L. Moiseev, M. Selim Ünlü, A. K. Swan, B. B. Goldberg, and C. R. Cantor, "DNA conformation on surfaces measured by fluorescence self-interference,” Proc. Natl. Acad. Sci. USA 103, 2623-2628 (2006).

17. A. K. Sharma and B. D. Gupta, "On the sensitivity and signal to noise ratio of a step-index fiber optic surface plasmon resonance sensor with bimetallic layers," Opt. Commun. 245, 159 169 (2005).

18. J. Homola, I. Koudela, and S. Yee, "Surface plasmon resonance sensors based on diffraction gratings and prism couplers: sensitivity comparison," Sens. Actuators B 54, 16-24 (1999).

19. B. Rothenhäusler and W. Knoll, "Surface-plasmon spectroscopy," Nature 332, 615-617 (1988).

20. X. Ku, Z. Tang, Z. Fan, and J. Shao, "Effect of chromium intermediate layer on properties of silver coatings," Opt. Eng. 43, 971-974 (2004).

21. D.-K. Qing and G. Chen, "Enhancement of evanescent waves in waveguides using metamaterials of negative permittivity and permeability," App. Phys. Lett. 84, 669-671 (2004).

22. N. Bassil, E. Maillart, M. Canva, Y. Levy, M. C. Millot, S. Pissard, R. Narwa, and M. Goossens, "One hundred spots parallel monitoring of DNA interactions by SPR imaging of polymer-functionalized surfaces applied to the detection of cystic fibrosis mutations," Sens. Actuators B 94, 313-323 (2003).

23. E. Maillart, K. Brengel-Pesce, D. Capela, A. Roget, T. Livache, M. Canva, Y. Levy, and T. Soussi, "Versatile analysis of multiple macromolecular interactions by SPR imaging: application to p53 and DNA interaction," Oncogene 23, 5543-5550 (2004). 